\title{
Understanding pain and pain management in elderly nursing home patients applying an interprofessional learning activity in health care students. A Norwegian pilot study
}

\author{
Elin Damsgård, RN, PhD, Associate professor ${ }^{1}$; Hege Solgård, PT, MS, Lecturer ${ }^{1}$; Karin Johannessen, RN, MS, \\ Lecturer $^{1}$; Katrine Wennevold, MD, Lecturer²; Gunnvald Kvarstein, MD, PhD, Professor ${ }^{3}$; Gunn Pettersen, \\ Sociologist, PhD, Associate Professor ${ }^{1}$; Beate Garcia, Pharmacist PhD, Associate professor ${ }^{4}$ \\ ${ }^{1}$ Department of Health and Care Sciences, ${ }^{2}$ Department of Community Medicine, ${ }^{3}$ Department of Clinical Medicine, \\ ${ }^{4}$ Department of Pharmacy, UiT/The Arctic University of Norway
}

\begin{abstract}
Pain is common among elderly patients in nursing homes; however, pain assessment and treatment are inadequate. Interprofessional treatment is recommended, and consequently interprofessional education in pain management is necessary. This pilot project aimed to describe how two interprofessional groups of students approached pain management in two nursing home patients. We formed two teams comprising one student from the nursing, physical therapy, pharmacy, and medical educations. Each team spent one day examining a patient with chronic pain at a nursing home and they developed pain management plans. We collected data through video recording during teamwork before and after examining the patient and field notes during the patient examination. We analysed the video-recordings applying the seven-step model including 1) viewing the video data, 2) describing the video data, 3) identifying critical events, 4) transcribing, 5) coding, 6) constructing storyline and 7) composing a narrative. Field notes supplied the transcripts. Both teams succeeded in making a pain management plan for their patient. The common examination of the patient was crucial for the students' approaches to pain management and changed their pre-assumptions about the patients' pain. By sharing knowledge and reflecting together, the students reached a common consensus on suggestions for management of the patients' problems. Interprofessional collaboration fostered enthusiasm and a more holistic pain management approach. However, students' lack of knowledge limited their understanding of pain. Knowledge of pain management in nursing home patients and the practice of interprofessional cooperation should be included in pain curricula for health care professionals.
\end{abstract}

Keywords: Pain, pain management, interprofessional education, nursing home 


\section{Introduction}

Health personnel caring for elderly nursing home residents experience several challenges related to the residents' poor health status. Chronic pain affects as many as $80 \%$ of institutionalized elderly patients (Chipchase, Allen, Eley, McAllister, \& Strong, 2012; Helme \& Gibson, 2001). Furthermore, chronic pain among nursing home residents is associated with reduced quality of life (Torvik, Kaasa, Kirkevold, \& Rustøen, 2010). Concurrent dementia may complicate pain management and increase the challenges for caregivers. A previous study indicated that nursing home residents with pain, especially those with dementia, often receive suboptimal treatment (Achterberg et al., 2013).

The multidimensional nature of pain includes biological, psychological, and social aspects; therefore, an interprofessional treatment approach to pain management is recommended (Gatchel, Peng, Peters, Fuchs, \& Turk, 2007). Accordingly, interprofessional teamwork is emphasized to pre-licensure health care professionals as one of the core principles in pain assessment and management (Fishman et al., 2013). Interprofessional education (IPE) is defined as an occasion when two or more professions learn with, from, and about each other to improve collaboration and quality of care (Centre for the Advancement of Interprofessional Education; CAIPE). IPE learning activities increase student knowledge of pain management, as well as their understanding of the benefits and necessity of working together (Carr, Brockbank, \& Barrett, 2003; Hunter et al., 2008). An important consideration for student satisfaction appears to be "real patient pain" experiences being included in the teaching activities (Clark, 2006; Hadjistavropoulos et al., 2015).

Despite a growing understanding of the necessity of pain education and interprofessional collaboration in pain management, IPE receives relatively minimal attention in undergraduate health care curricula (Carr \& Watt-Watson, 2012). Leegard, Valeberg, Haugstad, \& Utne (2014) demonstrated that there are no common pain curricula across six different Norwegian health care professions. In addition, there is considerable variation in the emphasis and organization of Norwegian pain education (Leegaard et al., 2014). Thus, pain knowledge may be inconsistent among Norwegian health care students, and pain management in Norwegian nursing homes may be inadequate. Furthermore, health care professionals in Norwegian nursing homes typically lack knowledge regarding pain assessment in the elderly and people with dementia (Torvik, Nordtug, Brenne, \& Rognstad, 2015), which emphasizes the need to focus on pain education. 
At the University of Troms $\emptyset$ (UiT), the Arctic University of Norway, IPE is a cornerstone principle in educating health care students. The present article reports a pilot project that aimed to improve student comprehension of pain in elderly nursing home patients. We assembled interprofessional teams of IPE-naïve students from different health care professions, in a real clinical setting; we asked the teams to assess pain and provide a pain management plan for elderly patients with pain, based on their present knowledge. We wanted to determine their existing knowledge and management strategies, thus providing a foundation for further interprofessional student pain management activity implementation. The educational aim was to develop students' shared understanding of pain and pain management and for them to achieve mutual understanding regarding each profession's contributions. The focus of this article is to describe how IPE-naïve interprofessional student teams approach pain management in elderly nursing home patients. Knowledge gained from this pilot project will be important for implementation of pain education in pre-licensed health care students.

\section{Material and methods}

\section{Setting}

This pilot project was a collaboration between UiT, the Arctic University of Troms $\varnothing$ (educational body), the University Hospital of Northern Norway (UNN), and the primary care sector of Troms $\emptyset$ Community (practice arena). The study was conducted at a community nursing home in Troms $\emptyset$, in February 2015. The nursing home is a clinical practice arena for third year nursing students every spring semester. The project group comprised educators and researchers from the schools of nursing (ED, KJ), physical therapy (HS), pharmacy (BG), and medicine (KW).

\section{Participants}

Two students (seven women, 1 man) from each of the following four health care fields participated: nursing ( $3^{\text {rd }}$ year), physical therapy $\left(3^{\text {rd }}\right.$ year $)$, pharmacy $\left(3^{\text {rd }}\right.$ year $)$, and medicine (one $3^{\text {rd }}$ year and one $5^{\text {th }}$ year student). We formed two teams of four students, including one student from each profession. The nursing students were included based on their clinical practice in the nursing home. The physical therapy students were included as part of their regular clinical practice studies in community service and the hospital (UNN). The medical students and the pharmacy students volunteered at their instructor's request. Only one of the medical students $\left(5^{\text {th }}\right.$ year) had clinical practice experience. 
The nursing students, in collaboration with clinical supervisors at the nursing home, selected two appropriate patients with pain. Both patients were female and in their 80s, with multiple diagnoses; one patient had dementia.

\section{Interprofessional learning activity}

We initiated the project period with a one-day introductory seminar, which included providing information about the project, a discussion of pain, and introducing students to each other. Other than the seminar, the students did not receive any training or education in pain management in addition to their respective curricula. The week following the introductory seminar, both teams met at the nursing home on different days. Before the teams met, the nursing students had collected relevant patient information and sent it to their respective teams.

We asked the teams to assess and evaluate their patient's pain, develop a pain management plan, and write a final report that included the management plan. We arranged a room at the nursing home for the teams to work from 8 am to $4 \mathrm{pm}$. The teams organized their work themselves, including planning their patient meetings. Educators from the project group (KJ and $\mathrm{KW}$ ) were present as IPE supervisors, and were ready to intervene if necessary. The nursing students' clinical supervisors were available for additional information throughout the day.

\section{Data collection}

This study was inspired by an ethnographic approach (Creswell, 2013); however, we applied nonparticipant observation using video recordings to overcome the subjectivity that results from having only one observer (Caldwell \& Atwal, 2005). The teams were videotaped while they were working in the allocated room, both before and after patient meetings. The video camera was placed in the corner to capture images of all four team members and recorded continuously throughout the discussions. In addition, we placed an audio recorder on the table to ensure good quality audio recordings of their discussions. The video and audio recordings started when the students first arrived in the morning, stopped during lunch and when students went to examine the patient, started again when the students returned from their examinations, and concluded at the end of the day. The recordings had total durations of $2 \mathrm{~h} 23 \mathrm{~min}$ and $2 \mathrm{~h} 43 \mathrm{~min}$. To protect these vulnerable patients and preserve confidentiality, we did not videotape patient examinations; however, the IPE supervisors wrote field notes. 


\section{Data analyses}

We used the video recordings as the basis for analyzing the students' discussions, supported by the audio recordings and field notes from the patient examination. The videos provided information regarding conversation content, nonverbal communication, interactions between students, and actions during the discussions. The field notes from the examination served as descriptive information regarding the team meetings with the patients. We analyzed the video data by applying a revised version of the analysis model presented by Powell, Francisco, \& Maher (2003), which was originally developed for use in mathematics education. The analysis method comprises seven steps: 1) attentively viewing the video data, 2) describing the video data, 3) identifying critical events, 4) transcribing, 5) coding, 6) constructing a storyline, and 7) composing narrative (Powell et al., 2003).

Step 1: We watched the video recordings and read the field notes from team supervisors before discussing our first impressions and comprehension (ED, $\mathrm{HS}, \mathrm{KW}, \mathrm{KJ}$, and $\mathrm{BG})$. We wanted to reduce subjectivity in interpretation and enhance reliability by watching the videos as a group (Caldwell \& Atwal, 2005).

Step 2: We discussed content from the teamwork sessions and patient examinations. At this point, we did not seek consensus, but rather we discussed perceptions of student interactions, both in relation to pain and pain management and also in relation to interprofessional teamwork.

Step 3: We identified video segments (1 h 75 min total) showing students directly or indirectly discussing pain, pain management, or the patient's pain related to other phenomena, using the wording "pain" or clearly referring to the pain experience (ED and HS).

Step 4: An independent person not directly involved in the research project transcribed the video recordings.

Steps 5 and 6: From the transcripts, which were supported by the field notes and reviewing the video segments, we identified sections and phrases relevant to our research questions, coded them, and reached a joint understanding of themes to form the storylines (ED, HS, KJ, and BG). Reviewing video recordings and data, and discussing alternative interpretations, were essential components of the analysis.

Step 7: Before composing the narratives, we read the full transcripts from the original videos again (ED) and discussed the final storyline (ED, HS, KJ, and BG). 


\section{Ethics}

One patient gave written consent to participate in the study, while the next of kin consented on behalf of the other patient. All students gave written consent to participate in the study. The Norwegian Centre for Research Data (NSD) approved the study.

\section{Results}

We identified four overall themes that were common for both teams with regard to understanding pain and approaching pain management: 1) becoming familiar with the patient, 2) that meeting the patient guides management approaches, 3) searching for explanations and pain relief, and 4) sharing knowledge and developing agreement.

\section{Theme 1: Becoming familiar with the patient}

The initial team meetings included the nursing students introducing the patients and their pain characteristics, and the other team members asking relevant questions.

In Team I, the nursing student described a patient with dementia, who had multiple painful fractures and physical immobility. On several occasions, the nursing student had found the patient weeping in the corridor, and she reported that the patient could not lift her arm. She said, turning to the physical therapy student, "but, if it is because of pain or...she (the patient) does not tell." She continued, "She gets [paracetamol] after physical therapy because she has a lot of pain..." The physical therapy student grimaced and took a note before looking at the nursing student and asking, "How often does she get physical therapy?"

During this interaction, the patient's physical therapy related pain was established, which seemed to concern the physical therapy student. The nursing student also expressed some uncertainty regarding the patient's pain, despite her regime of pain medication after physical therapy. The discussion revolved around the patient's situation, her sleeping problems, and the drowsiness that followed. Her dementia was addressed as a communication problem, but the team did not discuss how the dementia could impact her pain expressions, pain assessments, or medications. However, they agreed that the use of a numeric rating scale for pain assessment would not be useful for this patient.

The physical therapy student and the medical student both planned to examine the patient's painful shoulder. The pharmacy student was interested in an overview of the patient's pain medication, and concluded, "I do not know exactly what kind of pain she has, but if the effect of paracetamol is 
good, hmm, then you should stay on this level." However, the nursing student could not give an exact report on the efficacy of this medication for the patient. The student group agreed that it is upsetting for the patient to be alone and immobilized, and that it was important to improve her functionality.

In Team II, the nursing student reported that the patient had severe pain when walking, but no pain when sitting down. Addressing the team, she further reported, "I have not succeeded in making her elaborate more about where it [the pain] is situated and how the pain is... I think we can discuss that later on today."

In this way, the nursing student directed the teamwork and collaboration. The team continued their approach by discussing possible origins of the patient's pain. Even though the nursing student reported that the patient experienced anxiety, the team did not include this in their discussion at this point. After a long discussion, the physical therapy student said, "I think that first of all we must get to know her." The other students looked at the physical therapy student, who continued, "We have these usual questions...about social issues, function, ADL [activities of daily life] in relation to pain." The nursing student nodded in agreement, saying "yes". The medical student folded her arms, and leaned back in her chair, looking at the physical therapy student, who continued, "Related to her pain... I think that is perhaps the most important." She held up some papers, showing them to the team and said, "And I brought this one [a body map] and I also have such a grading scale." The grading scale was a numeric pain assessment tool for scoring the patient's own perception of pain on a scale from zero (no pain) to ten (worst possible pain). The nursing student looked at the physical therapy student and pointed at the papers, saying, "And it is very important to ask her to be 100\% honest as she is the kind of person who would say 4 when it is really a 10 [regarding the pain scale]."

At this time, the physical therapy student seemed to have established an agenda for the examination, and the nursing student, referring to the patient's personality, instructed the team to expect modesty when the patient reported her pain. This episode became a turning point for the team approach, as they then agreed on their need to see the patient. For both teams, this early phase of pain assessment without knowing the patient or the context seemed to be somewhat confusing. Neither of the groups formulated a joint structured pain assessment plan before meeting the patient.

Theme 2: Meeting the patient guides the pain management approach 
When Team I arrived in the patient's room, the nursing student and the medical student started the conversation with the patient by asking several consecutive questions. The patient appeared to have difficulty answering all of the questions, and said she had to go to the bathroom. The physical therapy student intervened, and she carefully explained to the patient that she wanted to help her out of bed while letting her manage as much as possible herself. She assisted the patient out of bed without the help of a lift and helped her walk to the restroom, supported only by a walker. The other students watched this, with the nursing student looking very surprised. During the restroom visit, the patient became more alert and started talking about a dinner party with her family the day before. After this, the physical therapy student appeared to be the leader of the student group during the continued examinations of her stomach and painful shoulder. The physical therapy student and the medical student both found indications of shoulder pain, as the patient expressed discomfort when lifting her arm.

In Team II, the patient was sitting in a chair when the team arrived in her room. The nursing student introduced the team, and then stepped aside. The patient primarily addressed the medical student, who seemed to take a leadership role during the examination. The team listened to her discuss her pain, and asked relevant questions. The team asked her to rate her pain, and emphasized the importance of being honest, according to the nursing student's instructions. The patient rated her pain as ten, meaning the "worst possible pain." The team subsequently asked the patient to walk from the chair to the bed. The patient indicated severe leg pain during this walk and was only able to walk a short distance. The medical student and physical therapy student continued their examination of the patient in bed, but had challenges ascertaining pain in areas including the knees, hips, and muscles.

Meeting the patients became important for both teams in their subsequent approaches. In Team I, the patient's cognitive and physical functions were better than the students had expected. In Team II, the patient's severe pain was unexpected, and resulted in the students interrupting their examinations during activity. The team dynamics were also affected by meeting the patients. In Team I, the physical therapy student stepped forward as a team leader. In Team II, the medical student became the leader during the examination, which also contributed to her being a leader in the discussions afterwards.

After meeting the patients, the students appeared to be emotionally affected by the patients' pain and circumstances, as expressed by the physical therapy student in Team II, who said, "I think we 
must write [in the report] that she has courage and willpower... and I think, regarding the pain, that she says there is nothing to be done about it. But there is!" The pharmacy student added spontaneously, "Yes, agree! Mmm.. I hope there is!"

Theme 3: Searching for explanations and pain relief options

After examining the patients, each team gathered to summarize, make a pain management plan, and prepare their reports. For both teams, the meeting was initially confusing.

In Team I, the nursing student turned to the supervisor, saying, "I do not think her pain was very evident..." The physical therapy student looked down at the table, nodding, and said, "No, it wasn't." The physical therapy student, the nursing student, and their supervisor reflected on what happened during the examination, and the other students listened to the discussion. At one point, the supervisor turned directly to the nursing student and asked, "How was it [the care situation] today compared to how it normally is? You have observed her [the patient] before. Do you think there was anything different?" The nursing student (thinking) answered, "I think she [the patient] really is the kind of person who likes to be in contact with other people...I once experienced how she was when she was in the restroom and I waited outside with my supervisor. Then she started weeping and said she wanted to hear what we talked about." The other students were sighing, expressing their sympathy with the patient, and the supervisor nodded, saying "Yes, exactly." The medical student then faced the nursing student and stated, "Then we are back at getting her out of the ward."

This episode seemed to be a turning point for Team I, as they redefined the patient's pain as primarily associated with lack of physical and social activity. The team subsequently engaged in a discussion about improving her social and physical activity, including how to manage her drowsiness, which was a barrier to activity.

The physical therapy student and the nursing student both turned to the pharmacy student, who then confirmed that medication could be influencing the patient's sleep, saying, "[She] takes that medication [melatonin]. It can also have an adverse effect. Can cause sleeping problems ...try not to give it." The medical student agreed, and added, "It does not seem to have an evident effect, so why should she use it?"

This interaction illustrates how pre-existing knowledge and experience are crucial for developing the treatment approach; the physical therapy student had previous experience with dementia care and the nursing student had in-depth patient knowledge. Despite observing a painful shoulder 
during the examination, the students focused their pain management approach on social and physical activity, in addition to discontinuing medication associated with drowsiness and continuing the pain medication.

Team II initially struggled with summarizing and understanding their patient's pain. The medical student, who was silent and withdrawn before meeting the patient, was very engaged in finding the reason for the patient's pain after meeting her. She suggested different diagnoses, and the team discussed whether any of these were relevant to the patient. The supervisor interjected, to help the students assess their data. She leaned forward and addressed the group, saying, "Have you discussed her numbness, which you describe?" The medical student responded, "That is interesting to sort out. Could be that she has a polyneuropathy." Looking at the nursing student, she added, "I wonder if that was the word you were looking for a while ago?" referring to a previous episode where the nursing student did not find the word for a diagnosis she was thinking about. "Yes! That's it!" the nursing student said eagerly. "I have seen this once before... and that lady [the previous patient] reminded me quite a lot about her [the present patient] regarding touch." The medical student then replied, "What is interesting here is that it is both painful and numb. That combination... I, at least, need to go home and study to understand what this is, " clearly allowing the team to understand her lack of knowledge.

The patient meeting was also important to Team II's approach to pain management; however, they did not achieve a complete understanding of the patient's pain. The team was searching for explanations, although they probably did not have the necessary knowledge to understand the patient's pain.

Theme 4: Sharing knowledge and developing agreement

After meeting the patients, knowledge sharing and professional discussions increased. The team meetings with the patients, including examining them together, appeared to increase their engagement, extend their discussions, and consequently promote knowledge sharing. The nursing students' previous observations and familiarity with the patients were fundamental factors, both for understanding the patients' pain and for influencing discussions regarding pain management. The pharmacy students shared knowledge of medications, including drug interactions, adverse drug reactions, and specific problems with drugs among elderly patients. The physical therapy students shared knowledge regarding improvements to patients' physical function, and how to support the 
patient resources. The medical students shared knowledge of relevant diagnoses and diagnostic measurements, and engaged in the general discussions of the patients' circumstances.

One example of knowledge sharing was when the physical therapy student in Team I suggested that the patient should get up by herself and walk. The nursing student expressed reluctance and uncertainty about helping the patient out of bed without technical aids. The physical therapy student then demonstrated how she would safely help the patient out of bed. The nursing student assumed the role of the patient while the physical therapy student demonstrated a technique for assisting the patient from a sitting to a standing position. The other students watched silently. Another example, from Team II, is the team's discussion of their observations related to the patient's sensitivity to touch. The nursing student turned to the physical therapy student, placed her hand on her shoulder, and said, "When I was just stroking [her leg], she felt tenderness, and that is...[thinking, and turning to the medical student] then it is quite serious." The medical student nodded, looking back at the nursing student and saying, "Yes, I also thought that was quite special. It was tender, and a bit numb. I thought maybe there is something going on in her spine. However, I do not know the tests to examine that." The pharmacy student then looked up from her papers, looked at the medical student and asked, "What do you think can be going on in her spine?" The medical student said, as she illustrated with her hands, "She has previously had a fracture. There could be something [thinking] pressing [her spine] somewhere." The pharmacy student stated, “Yes. Yes...[thinking] she has been on cortisone for a long time ... it is an immunosuppressive...it will impair her body's own defense system ...long term use is not good." The students eventually agreed that their patient's pain was likely not understood and required further examinations. The medical student suggested a magnetic resonance imaging scan, while the nursing student worried that it would be too exhausting for the patient. All students engaged in this cost-benefit discussion.

These episodes illustrate how team knowledge sharing and common reflections on their observations brought the students closer to an understanding of the patients' pain, including possible solutions. They also illustrate how meeting patients together as a team and sharing reflections provided an opportunity to complement each other's knowledge, even when all members were not active during patient meetings. Regarding pain management strategies, both teams suggested improved technical assistance, increased opportunities for physical and social activity, and medication changes. In Team II, the students also suggested heat treatment for pain relief. None of the groups suggested further pain assessments using standardized measures. 


\section{Discussion}

In this pilot study, we reported the approaches of two interprofessional health care student teams comprising nursing, physical therapy, medical, and pharmacy students, to pain management in nursing home patients with pain.

To understand the complex and subjective experience of pain, healthcare professionals require knowledge of a broad range of health problems. Such knowledge can be acquired through "situated learning" or learning and practice in authentic contexts (White, 2010). An important component of learning in practice is "reflection in action", such as learning from unexpected events (Kaufman \& Mann, 2010). In our study, unexpected situations during the patient meetings and during the team reflections provided valuable learning. Team I was surprised when the patient's functional level was better than expected, and they consequently navigated treatment planning in a different direction than planned. Team II went from being very eager to help with the patient's pain to accepting that they would not be able to do so without increased understanding of the pain origins. Adapting to unexpected events may be an advantage of real life learning.

Lack of knowledge was a barrier for both teams to fully understanding pain and providing pain management strategies. For Team I, the patient's dementia was especially challenging. None of the students on this team expressed knowledge of communication with a person with dementia, despite that dementia is included in both nursing and physical therapy curricula. Torvik et al. (2015) concluded that the use of pain assessment tools in Norwegian nursing homes appears to be deficient, which the present study also supports. Students on Team I had no knowledge of assessment tools for persons with dementia. The lack of knowledge was also apparent in Team II, since they did not consider the patient's anxiety diagnosis when discussing pain causes and pain relief. Since we clearly identified knowledge gaps, we believe there is a risk that students' lack of experience may jeopardize patient safety and proper treatment.

Despite these limitations, the student teams successfully discussed the patients' pain and suggested pain management plans. Meeting the patient was crucial for both teams. Interestingly, the pharmacy students, who did not have any previous clinical experience, also engaged in discussions regarding non-pharmacological approaches to pain management. We believe this supports the contention that meeting a patient in pain is important to understanding and engaging in holistic pain management. As previously identified, a patient's circumstances related to pain provide valuable insight and 
impactful learning for health care professionals (Carr, Worswick, Wilcock, Campion-Smith, \& Hettinga, 2012; Wood, Eccott, \& Bainbridge, 2013). Real life learning in clinical settings is generally valued both by students and by health care workers (Carr et al., 2012; Freeth et al., 2001; Reeves, Freeth, McCrorie, \& Perry, 2002), specifically the real life experience, responsibility, autonomy, and team reflections (Freeth et al., 2001; Reeves et al., 2002). These previous findings correspond with our analyses of the student interactions in the present study.

Effective communication and role understanding are highlighted as core competencies in IPE (Suter, Arndt, Arthur, Parboosingh, Taylor, \& Deutschlander, 2009). However, differences in knowledge and attitudes towards pain and pain management among health care professionals and students may challenge the development of these core competencies (Ali \& Thomson, 2009). In the present study, professional knowledge and perspectives became evident through team discussions, where both knowledge sharing and learning occurred as a result of pain management as a common task. Interprofessional skills developed simultaneously. This method of learning corresponds to a socio-cultural learning perspective, which is central to IPE (Hean, Craddock, \& O’Halloran, 2009).

The student positioning and learning from each other observed in the present study is consistent with findings by Lumague et al. (2006). This previous study also found that nursing students emphasized the importance of their patient interactions, which enabled them to communicate the patient's status and concerns to the other team members. Another similarity is that the physical therapy student shared knowledge of safe patient mobilization, and appreciated both medication information from the pharmacy student and updates on patient status from the nursing student (Lumague et al., 2006). In addition, we identified that the pharmacy students educated the other team members, explaining how medications could interact with each other and have side effects. This finding was not reported by Lumague et al. (2006). In a study by Reeves et al. (2002), nursing, physical therapy, and occupational therapy students found some discussions regarding patients too medically oriented, while medical students expected experiences that were more professionspecific (Reeves et al., 2002). This divergent perception among health care professionals has also been observed in other studies, and may be related to traditional hierarchical power relations (Whitehead, 2007) and differences in communication styles (Foronda, Macwilliams, \& McArthur, 2016). In the present study, we observed no indications of inequity or hierarchical structures during the team work. One reason for this difference may be the cultural context. In Norway, equity and 
non-hierarchy is valued, including in professional and academic settings. Our study indicates that it is important for students to learn interprofessional skills before hierarchical power relationships form. This skill development may benefit patients, health care workers, and the health care system.

\section{Ethical and methodological considerations}

The university supervisors, who were one medical doctor and one nurse, both had a special interest in pain management; however, they were not experts. Under these circumstances, the patients and their families may have had unrealistic expectations regarding patient treatment. Whether information was correctly provided, and whether it was understood by the people involved must be considered. One of the patients had dementia, which was a challenge regarding both informed consent and examinations. However, her daughter, who had consented on her behalf, and the supervisor were in room during the examination. The students' lack of knowledge limited their abilities to conduct appropriate assessments and make optimal suggestions. However, the nursing staff were very involved in the project, which mitigated potential ethical risks. The teams presented their reports to the staff and the nursing home physician, who gave them feedback and followed up on some of their suggestions.

The primary strength of our study is the use of video recordings, which enabled us to repeatedly assess the team communicative interactions (Caldwell \& Atwal, 2005; Powell et al., 2003). Caldwell et al. (2005) state that selective bias and memory limitations can be minimized by using video recordings, compared to direct observational studies (Caldwell \& Atwal, 2005). Furthermore, video recording improves the credibility of the research design, since other researchers can also view and interpret the observations (Caldwell \& Atwal, 2005). We considered the possibility that our personal knowledge of our respective students influenced our analyses. However, we believe that the team approach counteracted any potential bias, as different understandings and perspectives were discussed and interpretations were clarified, similarly to methods discussed by Powell et al. (2003). In addition, our analysis was itself interprofessional, which we believe is a strength of our study, and which increases the credibility of our results.

There are some limitations to our study. As described in the material and methods section, the university departments recruited students using different processes. Consequently, student motivation and engagement may have differed. In addition, videotaping may itself introduce a bias in how interactions occur when team members are aware of the video camera. However, habituation may also develop (Caldwell \& Atwal, 2005), which we believe was the case for our 
student teams. Finally, the medical doctor was present only during the first analyses, which may have influenced our understanding of the contributions and roles of the medical students.

Implications for nursing practice, education, and research

The roles of nurses in interprofessional pain management are diverse, and include coordinating the team, providing patient information to the team, and conducting pain assessments (Peng et al., 2008). As demonstrated in this pilot project, nursing student observations and familiarity with the patients were fundamental to the teams' approaches to pain management. Our pilot project provided an opportunity for the students to practice their professional roles in an authentic setting, with patients with complex pain. Feedback and discussions with these students from varied health care professions demonstrated the importance of systematic observations and pain assessments. Based on the findings of this pilot project, we believe that this interprofessional approach may be suitable for widespread implementation in the curricula of healthcare students in a variety of fields. Pain in elderly patients is a significant societal challenge, which is relevant to all health care students and may be ideally addressed through student collaborations. However, some refinements are necessary. We recommend that a more focused plan for student work should be developed to facilitate their preparedness to meet the patient. In addition, the students should have access to patient data, pain measurement tools, and relevant literature at an early stage in the collaboration. Furthermore, trained IPE supervisors who are familiar with pain education, as well as supervisors from each profession involved, should be available to support and guide the student discussions. We found that real life settings are unpredictable and may be confusing for the students. However, the reality and autonomy appear to be valuable learning experiences (Carr et al., 2012). Balancing the chaos and unpredictability of a real life setting with a more structured approach will be addressed by future work on this project. Inclusion in the curricula and organizing student teams was challenging, as also reported in other studies (Wood et al., 2013). The present pilot study was conducted on one day for each team. This type of short and well-defined experience may be easier to implement as a component of a full curriculum.

\section{Conclusions}


The present study indicates that IPE-naïve students in teams from different health care professions can collaborate and learn from each other when working with elderly nursing home patients with pain. Meeting the patients was crucial for understanding, engagement, and a more holistic approach to pain management. However, the student teams lacked knowledge regarding complex pain and pain assessment in the elderly, in people with dementia, and regarding the association between anxiety and pain. In summary, this IPE project provided valuable experiences and had implications for interprofessional pain management by student teams. Knowledge of pain management in nursing home patients and the practice of interprofessional cooperation should be included in pain curricula for health care professionals.

\section{Acknowledgements}

This study was funded by the Joint Education Committee of the University Hospital of Northern Norway and UiT, the Arctic University of Norway. The funding sources did not participate in any aspect of the study or in the decision to publish the findings. We thank the participants for their contributions, and also the nursing home staff for allocating patients and accommodating project managers and students. We also thank Professor Madeleine Abranth Dahlgren for advice on analytic approaches.

\section{Conflicts of interest: none}




\section{References:}

Achterberg, W. P., Pieper, M., van Dalen-Kok, A., de Waal, M., Husebo, B. S., Lautenbacher, S., et al. (2013). Pain management in patients with dementia Clin. Interv. Aging (Vol. 8, pp. 1471-1482).

Ali, N., \& Thomson, D. (2009). A comparison of the knowledge of chronic pain and its management between final year physiotherapy and medical students. European Journal of Pain, 13(1), 38-50. doi:10.1016/j.ejpain.2008.02.005

Caldwell, K., \& Atwal, A. (2005). Non-participant observation: using video tapes to collect data in nursing research. Nurse researcher, 13(2), 42-54.

Carr, E., \& Watt-Watson, J. (2012). Interprofessional pain education: definitions, exemplars and future directions. British Journal of Pain, 6(2), 59-65. doi:10.1177/2049463712448174

Carr, E. C. J., Brockbank, K., \& Barrett, R. F. (2003). Improving pain management through interprofessional education: evaluation of a pilot project. Learning in Health and Social Care, 2(1), 6-17. doi:10.1046/j.1473-6861.2003.00038.x

Carr, E. C. J., Worswick, L., Wilcock, P. M., Campion-Smith, C., \& Hettinga, D. (2012). Improving services for back pain: Putting the patient at the centre of interprofessional education. Quality in Primary Care, 20(5), 345-353.

Centre for the Advancement of Interprofessional Education (CAIPE). Statement of purpose. Retrieved from https://www.caipe.org/ April 2017

Chipchase, L., Allen, S., Eley, D., McAllister, L., \& Strong, J. (2012). Interprofessional supervision in an intercultural context: A qualitative study. Journal of Interprofessional Care, 2012, Vol.26(6), p.465-471, 26(6), 465-471. doi:10.3109/13561820.2012.718813

Clark, P. G. (2006). What would a theory of interprofessional education look like. Some suggestions for developing a theoretical framework for teamwork training 1. Journal of Interprofessional Care, 2006, Vol.20 (6), p.577-589, 20(6), 577-589. doi:10.1080/13561820600916717

Creswell, J. W. (2013). Qualitative inquiry \& research design: choosing among five approaches (3rd ed. ed.). Los Angeles: Sage.

Fishman, S. M., Young, H. M., Lucas Arwood, E., Chou, R., Herr, K., Murinson, B. B., et al. (2013). Core Competencies for Pain Management: Results of an Interprofessional Consensus Summit (Vol. 14, pp. 971-981).

Foronda, C., Macwilliams, B., \& McArthur, E. (2016). Interprofessional communication in healthcare: An integrative review. Nurse Education in Practice, 19, 36-40. doi:10.1016/j.nepr.2016.04.005

Freeth, D., Reeves, S., Goreham, C., Parker, P., Haynes, S., \& Pearson, S. (2001). 'Real life' clinical learning on an interprofessional training ward. Nurse Education Today, 21(5), 366-372. doi:10.1054/nedt.2001.0567

Gatchel, R. J., Peng, Y. B., Peters, M. L., Fuchs, P. N., \& Turk, D. C. (2007). The biopsychosocial approach to chronic pain: scientific advances and future directions. Psychological Bulletin, 133(4), 581-624.

Hadjistavropoulos, H. D., Juckes, K., Dirkse, D., Cuddington, C., Walker, K., Bruno, P., et al. (2015). Student evaluations of an interprofessional education experience in pain management. Journal of Interprofessional Care, 2015, Vol.29(1), p.73-75, 29(1), 73-75. doi:10.3109/13561820.2014.917613

Hean, S., Craddock, D., \& O'halloran, C. (2009). Learning theories and interprofessional education: a user's guide. Learning in Health and Social Care, 8(4), 250-262. doi:10.1111/j.14736861.2009.00227.x

Helme, R. D., \& Gibson, S. J. (2001). The epidemiology of pain in elderly people. Clinics in Geriatric Medicine, 17(3), 417-431, v. 
Hunter, J., Watt-Watson, J., McGillion, M., Raman-Wilms, L., Cockburn, L., Lax, L., et al. (2008). An Interfaculty Pain Curriculum: Lessons learned from six years experience. Pain, 140(1), 74-86. doi:10.1016/j.pain.2008.07.010

Kaufman, D. M., \& Mann, K. V. (2013). Teaching and Learning in Medical Education: How Theory can Inform Practice. In (Ed) T.Swanwick Understanding Medical Education. Evidence, Theory and Practice (2nd ed., pp. 7-29). John Wiley \& Sons, Ltd, Oxford, UK.

Leegaard, M., Valeberg, B. T., Haugstad, G. K., \& Utne, I. (2014). Survey of Pain Curricula for Healthcare Professionals in Norway. Nordic Journal of Nursing Research, 34(1), 42-45. doi:10.1177/010740831403400110

Lumague, M., Morgan, A., Mak, D., Hanna, M., Kwong, J., Cameron, C., et al. (2006). Interprofessional education: The student perspective. Journal of Interprofessional Care, 2006, Vol.20(3), p.246253, 20(3), 246-253. doi:10.1080/13561820600717891

Peng, P., Stinson, J. N., Choiniere, M., Dion, D., Intrater, H., LeFort, S., et al. (2008). Role of health care professionals in multidisciplinary pain treatment facilities in Canada. Pain Research and Management, 13(6), 484-488.

Powell, A. B., Francisco, J. M., \& Maher, C. A. (2003). An analytical model for studying the development of learners' mathematical ideas and reasoning using videotape data. Journal of Mathematical Behavior, 22(4), 405-435. doi:10.1016/j.jmathb.2003.09.002

Reeves, S., Freeth, D., McCrorie, P., \& Perry, D. (2002). 'It teaches you what to expect in future...': interprofessional learning on a training ward for medical, nursing, occupational therapy and physiotherapy students. Medical Education, 36(4), 337-344. doi:10.1046/j.13652923.2002.01169.x

Suter, E., Arndt, J., Arthur, N., Parboosingh, J., Taylor, E., \& Deutschlander, S. (2009). Role understanding and effective communication as core competencies for collaborative practice. Journal of Interprofessional Care, 2009, Vol.23(1), p.41-51, 23(1), 41-51. doi:10.1080/13561820802338579

Torvik, K., Kaasa, S., Kirkevold, $\varnothing$. \& Rustøen, T. (2010). Pain and Quality of Life Among Residents of Norwegian Nursing Homes. Pain Management Nursing, 11(1), 35-44. doi:10.1016/j.pmn.2009.01.001

Torvik, K., Nordtug, B., Brenne, I. K., \& Rognstad, M.-K. (2015). Pain Assessment Strategies in Home Care and Nursing Homes in Mid-Norway: A Cross-sectional Survey. Pain Management Nursing, 16(4), 602-608. doi:10.1016/j.pmn.2015.01.001

White, C. (2010). A socio-cultural approach to learning in the practice setting. Nurse Education Today, 30(8), 794-797. doi:10.1016/j.nedt.2010.02.002

Whitehead, C. (2007). The doctor dilemma in interprofessional education and care: how and why will physicians collaborate? Medical Education, 41(10), 1010-1016. doi:10.1111/j.13652923.2007.02893.x

Wood, V., Eccott, L., \& Bainbridge, L. (2013). A Blended Active Learning Pilot: A Way to Deliver Interprofessional Pain Management Education. Pharmacy, 1(2), 218-227. doi:10.3390/pharmacy1020218 